performing a longitudinal experiment on 6 male and 6 female C57BL/ 6 mice which will result in VOM profiles for each mouse along the time course of 8 weeks of a mouse's life, also providing us with 'normal' inter- and intra-animal metabolite variation. Such data will allow calibration of future studies of colonic disease in humans through the induction of colonic disease in mice.

Disclosure of Interest None Declared.

\section{PTH-026 PREDICTORS FOR ALL CAUSE MORTALITY FOLLOWING CLOSTRIDIUM DIFFICILE ASSOCIATED DIARRHOEA}

doi:10.1136/gutjnl-2013-304907.514

1."S Thayalasekaran, 'J Cuthbertson, 'V Subramanian. 'Gastroenterology, Leeds Teaching Hospitals, Leeds, UK

Introduction Severe Clostridium difficile associated diarrhoea (CDAD) is an important nosocomial infection, often resulting in severe morbidity or death. The rates of CDAD have increased significantly in the last 2 decades, but predictors of outcome are poorly understood.

Methods A retrospective cohort study was performed in patients with a diagnosis of CDAD hospitalised at Leeds Teaching Hospitals NHS Trust (LTHT) between January 2011 and December 2011. The data on these cases was collected from electronic patient records and medical notes. Data collected included general demographics, underlying medical conditions, Horn Index, Charlson co-morbidity score, clinical and laboratory data, and the medical treatment given. Death due to any cause either during that hospital stay or within 30 days of discharge from hospital was the primary outcome. Severe CDAD was defined according to the UK Health Protection Agency (HPA) guidelines as WCC $>15 \times 10^{9} / \mathrm{L}$, or an acute rising serum creatinine (i.e. $>50 \%$ increase above baseline), or a temperature of $>$ $38.5^{\circ} \mathrm{C}$, or evidence of severe colitis (abdominal or radiological signs). Logistic Regression analysis was used to identify parameters associated with mortality. SPSS version 17 (IBM Corp, NY) was used to perform the statistical analysis.

Results There were 247 patients with a diagnosis of CDAD made in 2011 at LTHT of which 16 were wrongly coded, 5 were treated in the community, 12 had insufficient information in the notes and in 68 patients the medical notes could not be traced. A total of 170 episodes in 146 patients were finally analysed. There were 36 deaths in this group. Patients who were dead were older (mean age $78 \pm 12.9$ vs 76.6 \pm 17.6$)$. Independent predictors of mortality on multivariate analysis included age (OR 1.051, 95\% CI 1.009-1.095), Charlson comorbidity score of $\geq 3$ (OR 3.036, 95\% CI 1.209-7.622), Horn Index (Major or Extreme) (OR 4.725, 95\% CI 1.818-12.283), Severe CDAD (OR 3.454, 95\% CI 1.222-9.760) and in-appropriate treatment of severe CDAD with metronidazole as first line therapy (OR 4.642, 95\% CI 1.213-19.193). Factors not found significant included gender, prior use of antibiotics, PPI use, opioid use, prior episodes of $\mathrm{CDAD}$ and treatment with vancomycin.

Conclusion Predictors of all-cause mortality in patients with CDAD include older age, Charlson score $\geq 3$, Horn index $\geq 3$, severe CDAD as defined by the UK HPA and in-appropriate use of metronidazole in severe $\mathrm{CDAD}$. Patients with severe $\mathrm{CDAD}$ should not be treated with Metronidazole as first line therapy. Further prospective validation of these results is needed in a multicenter setting.

Disclosure of Interest None Declared.

\section{PTH-027 STOPPING PROTON PUMP INHIBITORS TO PREVENT CLOSTRIDIUM DIFFICILE: IS IT POSSIBLE AND DOES IT HELP?}

doi:10.1136/gutjnl-2013-304907.515

${ }^{1} \mathrm{M}$ Popescu, ${ }^{1} \mathrm{~K}$ Welch, ${ }^{2} \mathrm{R}$ Cunninghan, 1,"S Lewis. 'Gastroenterology; ${ }^{2}$ Microbiology, Derriford Hospital, Plymouth, UK
Introduction Hypochlorhydydria due to proton pump inhibitor (PPI) therapy is associated with increased susceptibility and propensity to relapse from infection due to $C$ difficile. The Health Protection Agency guidance advises review of PPI use in patients with CDAD. We have done a retrospective audit of patients with CDAD examining PPI use and any benefit achieved by stopping the PPI.

Methods All patients with CDAD between 2010-12 were reviewed by infection control nurses and advised the attending doctors to stop any PPI therapy where a clear indication was not obvious. We have retrospectively audited patient's relevant baseline characteristics, clinical outcome, length of hospital stay and PPI prescription at hospital discharge. In addition we followed patients up for 90days post discharge recording PPI prescription and further CDAD

Results 75 patients, 38 taking a PPI (mean age 77 stD) and 37 not taking a PPI (mean age $72 \mathrm{StD}$ ). No difference was seen for antibiotic use, co morbidity, immunosuppression, recent surgery, enteral feeding or dependency. At hospital discharge of those taking a PPI $12 / 38(32 \%)$ had died. The PPI was stopped in $17 / 26(65 \%)$, further $\mathrm{CDAD}$ occurred in 3 taking a PPI and 5 not. Of those not taking a PPI on hospital admission 16/37 (43\%) died by hospital discharge and at 90days 4 developed further CDAD.

Conclusion It was possible to stop PPI prescription in $65 \%$ of patients presenting with $\mathrm{CDAD}$. We found no evidence that CDAD was more severe in patients taking a PPI or that there was reduced recurrence of CDAD in patients who had stopped taking their PPI or had never taken it.

Disclosure of Interest None Declared.

\section{PTH-028 IS FLEXIBLE SIGMOIDOSCOPY EVER ENOUGH? AN AUDIT OF THE RATES OF PROXIMAL DISEASE DURING COLONOSCOPY}

doi:10.1136/gutjnl-2013-304907.516

1.*S Macdonald, 'S Radhakrishnan, 'E Seward. 'Gastroenterology, Whipps Cross Hospital, London, UK

Introduction There is debate about appropriate initial endoscopic investigation of patients with symptoms suggestive of colorectal cancer. Thompson (2008) proposed that for patients without iron deficiency anaemia or abdominal mass; Flexible Sigmoidoscopy (FS) should be first line with further colonoscopy only if significant distal colonic disease was detected. A proximal cancer miss rate of 1:500 patients over 60 years and 1:3000 below this age was reported. It was argued that this approach would significantly reduce costs and burden on patients.

However Lieberman (2000) reported $2.7 \%$ of asymptomatic patients screened with colonoscopy had proximal neoplasms without distal disease.

We have applied the Thompson criteria to our unit's colonoscopy data to establish the rate of disease, both neoplastic and other, proximal to the splenic flexure which would have been missed by performing $\mathrm{FS}$ alone.

Methods All colonoscopies performed in our unit over 2 months were examined and those which met the Thompson criteria for FS were included. The procedure and pathology reports were reviewed and the number with proximal but without distal disease, and type of pathology was established. Adenoma with low grade dysplasia (LGD) was the minimum classification for neoplasm.

Results 342 colonoscopies were reviewed and 200 met the Thompson criteria for FS. 17 (8.5\%) patients had proximal disease without distal disease, of which 9 (53\%) were neoplastic and $8(47 \%)$ had significant other disease ( $87.5 \%$ inflammatory). Of the 82 patients $>$ 60 years, it was found that $10(12.1 \%)$ had significant disease, of which $8(80 \%)$ were neoplastic (6 tubular adenoma with LGD, 2 tubulovillous adenoma with LGD). Of the $118<60$ years, $7(5.9 \%)$ had significant disease (85\% inflammatory) and 1 (15\%) neoplastic. Conclusion $4.5 \%$ of patients who would have had a FS using Thompson criteria were found to have a proximal neoplasm. This is 